CoRollary 2. If $\phi: H^{k}(A) \rightarrow H^{k}(\bar{A})$ is an isomorphism for $k \leqq n$ and univalent for $k=n+1$, and $\phi: H\left(H^{+}(X, A)\right) \rightarrow H\left(H^{+}(X, \bar{A})\right)$ is an isomorphism, then $\phi: H^{k}(\Gamma A) \rightarrow H^{k}(\Gamma \bar{A})$ is also an isomorphism for $k \leqq n$ and univalent for $k=n+1$.

\title{
REFERENCE
}

1. H. Cartan, Seminaire de topologie algébrique, E.N.S., 1950-1951.

University of California, Los Angeles

\section{ONE-PARAMETER TRANSFORMATION GROUPS IN THE PLANE}

PAUL S. MOSTERT

Very little is known about the action of a one-parameter group $R$ on two-space except when all orbits are circles, in which case the action is completely known [1]. In a forthcoming paper, A. Beck proves that any closed set can act as the set of fixed points for $R$. Hence, a very general description appears to be hopeless. However, here, we are able to prove the following result.

Theorem. Let $E$ be the plane and $R$ the real line acting on $E$ as a group of transformations without fixed points (i.e., no point is left fixed by all of $R$ ). If $E / R$ is Hausdorff, then $E$ is fibred as a direct product of $R$ and a cross sectioning line. Thus, $R$ is equivalent to a group of translations.

Proof. Let $x \in E$. Since $x$ is not fixed under $R$, there is a closed interval $[-a, a]=T$ about 0 in $R$, and an arc $C \subset E, x \in C$ but not an end point of $C$, such that $T^{2}(C)$ is a compact neighborhood of $x$ and the mapping $(t, c) \rightarrow t(c)$ is one to one from $T^{2} \times C \rightarrow T^{2}(C)$. That is, $C$ is a local cross section to the local orbits of $T^{2}[1]$. We shall show that $C$ is a local cross section for the orbits of $R$.

Suppose, on the contrary, that for some $z \in C$, there is an $r>a$ such that $r(z) \in T(C)$. Let $b$ be the greatest lower bound of such numbers. Then $b(z) \in-a(C)$, for if not, say $b(z)=t(c), t \in T, c \in C$, and $t>-a$, then there is a $t^{\prime},-a<t^{\prime}<0$, such that $t+t^{\prime}>-a$. Hence $\left(b+t^{\prime}\right)(z)=\left(t+t^{\prime}\right)(c) \in T(C)$. But $t^{\prime}<0$ so that $b+t^{\prime}<b$. By the choice of $b$, this implies $b+t^{\prime}<a$. Since this implies $b+t^{\prime \prime}<a$ for

Presented to the Society, April 19, 1957; received by the editors December 21, 1956. 
$t^{\prime} \leqq t<0, b=a$. That is, for some $t, 0<t<a,(b+t)(z) \in T(c)$. But this contradicts the fact that $T^{2}(C)$ is homeomorphic to $T^{2} \times C$. Thus $b>a$, and $b(z) \in-a(C)$.

Suppose $b(z)=-a(z)$. Then $[-a, b](z)=R(z)$ is a circle bounding a pre-compact region $A$ by the Jordan curve theorem. Hence, if $z \in A, R(z) \subset A$ since orbits cannot intersect. Then, for any $r \in R$, $r\left(A^{-}\right) \subset A^{-}$. Thus $r$ has a fixed point. For each $n$, let $x_{n}$ be a fixed point for $1 / 2^{n}$. Let $y$ be a limit point of the $x_{n}$ 's. Thus $R(y)=y$, contradicting our hypothesis that $R$ acts without fixed points.

Now we may assume $b(z) \neq-a(z)$. Let $[b(z),-a(z)]=$ $-a[(a+b)(z), z]$ denote the arc of $-a(C)$ joining $b(z)$ to $-a(z)$. Then $[-a, b](z) \cup[b(z),-a(z)]$ is a simple closed curve in $E$, and thus divides $E$ into two parts $A$ and $B$ one of which is pre-compact. Moreover, if $R_{+}=(0, \infty), R_{-}=(-\infty, 0), R_{+}(b(z))$ is contained in one, say it is $A$, while $R_{-}(-a(z))$ is contained in the other, for $R_{+}(b(z))$ cannot leave $A$ except by crossing $[b(z),-a(z)]$ since an orbit cannot cross itself. But $R_{+}(b(z))$ cannot leave $T(C)$ except by crossing $a(C)$. A similar argument establishes that $R_{-}(-a(z)) \subset B$. Moreover, if $z \in A$, and $r>0$, then $r(z) \in A$ since again $R(z)$ cannot cross $[-a, b](z)$, and cannot leave $T(C)$ through $-a(C)$. Hence, for $r>0, r\left(A^{-}\right) \subset A^{-}$. But $A^{-}$is a closed two-cell since it is bounded by a simple closed curve. Thus $r$ has a fixed point. Again find $x_{n} \in A^{-}$ such that $1 / 2^{n}\left(x_{n}\right)=x_{n}$. If $y$ is a limit point of $x_{n}$, then $R(y)=y$, a contradiction.

This proves that $C$ meets $R(x)$ in exactly one point for each $x \in T(C)$. Thus, $C$ is a local cross section for all of $R$. Since we can find a local cross section for each point of $E$, and since $E / R$ is Hausdorff, $E$ is a fibre bundle over $E / R$ with fibre $R$ and base space a connected one-dimensional manifold. Since $E$ is a principal fibre bundle over $E / R$, and $R$ is the line, there is a cross section $L$ of $E / R$ in $E$ such that the natural mapping $E \times R \rightarrow R(E)$ is a homeomorphism onto $E[2]$. Clearly $L$ must be a line.

\section{BIBLIOGRAPHY}

1. D. Montgomery and L. Zippin, Transformation groups, Interscience Publishers, 1955.

2. N. Steenrod, Topology of fibre bundles, Princeton University Press, 1951.

TUlane University 